\title{
The past is prologue: the profundity, productivity and progress of the early HBV years
}

\section{Harvey J. Alter}

Department of Transfusion Medicine, Warren Grant Magnuson Clinical Center, National Institutes of Health, Bethesda, MD, USA

Huge outbreaks of "serum" hepatitis in World War II led to intensive efforts to discover hepatitis viruses over the next two decades. These directed efforts better defined serum and infectious hepatitis, but failed to identify the causative agent. Where direction and intent failed, chance observation, an incorrect hypothesis and serendipity dramatically changed the direction of hepatitis research and set the foundation for blood screening, vaccine development, antiviral therapy and the realistic hope that over the next 1-2 generations, hepatitis B might be eradicated as a global endemic. The paradigm shifting event in hepatitis B virus research was the obscure and serendipitous finding of a precipitin line in agar gel that had unique staining characteristics. On a day, circa 1962, that seemed no more momentous at the time than any other day, the serum of an Australian aborigine precipitated with that of a multiply transfused patient with hemophilia; that precipitin failed to take up the lipid stain that was characteristic of the lipoprotein polymorphisms that were being studied in the laboratory of Baruch Blumberg on the NIH campus. The precipitin instead stained intensely red when the protein counter-stain, azocarmine, was applied. For a brief period the reactive protein was called the "red antigen" for this staining characteristic, but that name was supplanted when it was decided to name it after the country of origin of the protagonist and hence was born the designation, "Australia antigen." The relevance of that antigen was unknown at the time and certainly no thought was given to the possibility that it might represent a component of a hepatitis virus. Rather, because subsequent studies showed the antigen to be found in only $0.1 \%$ of healthy 
blood donors, but in $10 \%$ of patients with leukemia, it was postulated in the first paper to describe the Australia antigen that it might be related to a then hypothesized leukemia virus. Of note, of the three authors on that initial paper, none had a demonstrated interest or expertise in viral hepatitis at the time. Rather the lead author, Blumberg, was a geneticist, the second author, Alter, was a hematologist and the third author, Visnick was a technologist who six months later became a commercial airline pilot. Such are the elements of discovery and such is the importance of nondirected research. I was the first to observe this unique precipitin, but I remember no celebratory events and no dreams of Nobel prizes. It was just another day in the lab although it did set the course for all hepatitis B research to follow. This observation easily could have been abandoned as a minor finding of unknown significance, but Blumberg pursued it tenaciously. As a geneticist, Blumberg, by then working at the Institute of Cancer Research in Philadelphia, believed that the Australia antigen was inherited and that the associated gene might predispose to leukemia. Hence, he studied patients with a genetic predisposition to leukemia, namely patients with Down's syndrome. It was found that Down's patients had the same high prevalence of the antigen as patients with leukemia, thus seeming to substantiate the genetic-leukemia-linkage hypothesis. However, a subsequent study showed that the Australia antigen had a highly skewed distribution within the Down's population being found in approximately $30 \%$ of patients housed in large institutions, $10 \%$ of those in small institutions, $3 \%$ in those residing at home and, in none at the time of birth. Given the unhygienic conditions of mental institutions at that time, this suggested that the antigen, rather than being inherited, might be infectious in origin. However, there was still no clue as to which infection until a second serendipitous event. An investigator in the Blumberg lab, who had repeatedly tested Australia antigen negative, retested herself at the time she was becoming symptomatic from an episode of laboratory-acquired acute hepatitis. Her icteric serum tested strongly positive and the former "red antigen" had sudden new meaning as a probable component of a human hepatitis virus. This was further confirmed in other patients with hepatitis and in comparisons of ALT levels among Down's patients and dialysis patients who did or did not harbor the Australia antigen. Subsequent studies in other laboratories showed that the Australia antigen was specific for hepatitis B and that the antigen represented the envelope protein of a $42 \mathrm{~nm}$ particle first observed by Dane using immune electron-microscopy. This led to the renaming of the antigen as the hepatitis B surface antigen (HBsAg). Of great importance, it was observed that small spherical particles and large tubular forms consisted solely of surface antigen and that these incomplete virions outnumbered whole virions (Dane particles) by at least 1000-fold giving rise to the concept that isolated, non-infectious particles might serve as a raw vaccine. One cannot overemphasize the importance of this characteristic of HBV replication, namely the production of a huge excess of antigenic, but non-infectious particles. It was this massive antigenic load that allowed the agent to be detected by a technique as insensitive as agar gel diffusion. One could calculate that in an infection where there are typically 
$10^{6}$ to $10^{10}$ infectious virions $/ \mathrm{ml}$, incomplete virions would bring the antigenic mass to at least $10^{9}$ to $10^{12}$ particles per $\mathrm{ml}$ and hence into the sensitivity range of unsophisticated detection systems. This antigenic mass also allowed for early implementation of first generation assays to screen blood donors. Some institutions began testing donors by in-house agar gel diffusion systems as early as 1970 and in 1971 when commercial assays became available. By 1973 sensitive radioimmunoassys and enzyme immunoassays had been licensed. Using these more sensitive assays, retrospective testing of prospectively collected samples at NIH revealed that prior to HBsAg donor testing, only 25\%-30\% of transfusion-associated hepatitis cases were due to HBV infection and hence that an additional non-B agent must exist. By 1975 , prospective studies showed that transfusion-related HBV cases had been near eradicated though larger studies and mathematical models showed that antibody seroconversions in donors and recipients continued to occur. By serologic exclusion of HBV and later of HAV, it became apparent that a non-A, non-B agent was the major cause of transfusion-associated hepatitis. Thus, the Australia antigen also set the stage for subsequent studies that culminated in the discovery of the hepatitis $\mathrm{C}$ virus.

Although the prevention of transfusion-associated hepatitis was a dramatic outcome of Australia antigen/HBV discovery, it was surpassed in impact by the development of a novel HBV vaccine. Studies by Krugman at Willowbrook State School showed that his classic MS-2 virus was identical to HBV and that by boiling MS-2 containing plasma, it could be rendered non-infectious while retaining antigenicity and inducing strong antibody to HBsAg. This experimentally supported the concept that inactivated plasma could be employed as a first generation vaccine. In subsequent refinements, HBsAg particles were mechanically separated from infectious virions based on differences in size and density and residual virions inactivated by formalin and low ph. The efficacy of this early plasma-derived vaccine was demonstrated conclusively in studies by Szmuness and coworkers in a population of high risk men who have sex with men. This beautifully controlled and conducted study was crystal clear in its outcome. While new hepatitis B infections were unabated in controls, new infections in vaccinees were exceedingly rare after discounting early infections that were incubating at the time of vaccine administration. Indeed, the only cases that occurred were in the $5 \%$ of individuals who did not mount an antibody response after vaccination. The irony of this early vaccine story is that despite the proven efficacy and safety of the vaccine, few individuals chose to be vaccinated because the source material was plasma from high-risk individuals and because HIV infection was emerging in this same population at the that time. Hence, in the US, HBV vaccination did not take a strong foothold until Maurice Hilliman and associates at Merck developed a recombinant HBV vaccine. The development of this vaccine was a monumental achievement that has the potential to eradicate $\mathrm{HBV}$ infection if it can be made available at reasonable cost to the highly HBV endemic populations of Asia and sub-Saharan Africa.

In parallel with the US vaccine studies were equally remarkable studies by Beasley and associates in Taiwan. In a landmark investigation, Beasley et al. showed that the 
established $90 \%$ chronic carrier rate in the offspring of HBeAg-positive mothers could be reduced to $5 \%$ if neonates were given the combination of hepatitis B immune globulin (IBIG) and HBV vaccine. This extraordinary efficacy set the stage for routine HBsAg testing of pregnant women and the administration of HBIG and/or vaccine to their offspring and later to universal vaccination of all newborns in countries with the resources to do so. Beasley and coworkers also conducted another remarkable study in which they determined the cause of death in 22,707 Taiwanese government employees. Because there was a death benefit to be claimed by the families, case ascertainment was exceedingly high. Although only a small minority of the total population was HBsAg+ at initiation of the study, approximately one-third of the total deaths occurred in that minority. Remarkably, of 105 deaths in HBsAg+ subjects, 17 were attributed to cirrhosis and 40 to hepatocellular carcinoma (HCC). In contrast, of the 202 deaths in HBsAg negative subjects only 2 were due to cirrhosis and one to HCC. The relative risk for HCC in those HBsAg+ exceeded 200. Although many prior studies had shown a link between HBV and HCC, this study in Taiwan was the most convincing and provides strong evidence that an $\mathrm{HBV}$-preventing vaccine also serves as a cancer vaccine.

Thus within 20 years of the chance discovery of the Australia antigen: 1) a blood donor screening test was in place in all developed countries and served to reduce clinical hepatitis B and the HBsAg carrier state in transfusion recipients to near zero; 2) non-B hepatitis was recognized sequentially leading to the discovery of HCV and the near total eradication of post-transfusion hepatitis; 3) a hepatitis B immunoglobulin was prepared and shown to be effective in reducing materno-fetal transmission, in reducing the risk of needlestick exposure and, much later, in preventing HBV recurrence in transplanted livers; 4) highly efficacious plasma-derived, and later, recombinant $\mathrm{HBV}$ vaccines were developed and shown to provide long-term protection to all persons capable of mounting an anti-HBs response; 5) the high risk of materno-fetal transmission of HBV was convincingly demonstrated and shown to be prevented in $95 \%$ of neonates by the combination of HBIG and vaccine; and 6) the association between $\mathrm{HBV}$ and $\mathrm{HCC}$ was unequivocally established adding a new dimension to the value of universal vaccination and validating the concept of viralbased cancer vaccines. This is a remarkable sequence of events stemming from the chance finding of a precipitin line that stained the wrong way. It is a testimony to non-directed research, to observation and to perseverance. It is the stain heard round the world.

The last 20 years have seen remarkable refinements in our knowledge of HBV replication, virus-host interactions and the development of novel therapies as will be described in this text. However, the major events happened in the first 20 years and it will be difficult to match them for sheer excitement and for their implications on global health. 